\title{
Observations of the upper frequency cutoffs of the auroral kilometric radiation
}

\author{
J. Hanasz ${ }^{1}$, R. Schreiber ${ }^{2}$, H. de Feraudy ${ }^{3}$, M. M. Mogilevsky ${ }^{4}$, T. V. Romantsova ${ }^{4}$ \\ ${ }^{1}$ Space Research Centre, P.A.S., Torun, Poland \\ ${ }^{2}$ Nicolaus Copernicus Astronomical Centre, Laboratory for Astrophysics, Toruń, Poland \\ ${ }^{3}$ Centre d'Etude des Environnements Terrestre et Planétaire CNRS, Velizy, France \\ ${ }^{4}$ Space Research Institute, R.A.S., Moscow, Russia
}

Received: 24 July 1997 / Revised: 6 April 1998 / Accepted: 27 May 1998

\begin{abstract}
Intense auroral kilometric radiation (AKR) is being frequently observed with POLRAD from the Auroral Probe (Interball-2). Observations of the abrupt upper frequency cutoffs (UFCs) in the spectra of AKR are reported. The UFCs can be observed at a frequency range from 300 to $700 \mathrm{kHz}$, corresponding to AKR generation altitudes from approximately 4800 to $2100 \mathrm{~km}$, and are distributed in magnetic local time (MLT) hours similarly to the AKR events, with a maximum at $1 \mathrm{~h}$ MLT. The observed frequency extent of the UFCs is $\leq 12 \mathrm{kHz}$, and is often determined by the instrumental resolution $(4 \mathrm{kHz})$. It is suggested that the UFC may be associated with an abrupt switching on of the generation mechanism, when the electron density becomes sufficiently low inside a plasma depletion at an altitude where the ratio of $f_{p e} / f_{c e}$ crosses some threshold value. The steepness of the UFCs can imply a non-linear process of generation. The estimated distance of the e-folding field aligned wave amplification is between 3 and $8 \mathrm{~km}$. The UFCs are sometimes, though very seldom $(<10 \%)$, accompanied by narrow band (less than $4 \mathrm{kHz}$ ) "ridges" of radiation observed at the cutoff frequency. They are smoothly drifting in frequncy for several minutes. The power density of radiation in the "ridge" can be up to 2 orders of magnitude stronger than in the accompanying wide band emission of AKR. The "ridge" at UFC can imply either energy concentration at the source bottom, or focusing, if specific conditions for the escape of the radiation are assumed.
\end{abstract}

Key words. Magnetospheric physics (auroral phenomena; plasma waves and instabilities) . Radio science (magnetospheric physics)

\section{Introduction}

Interball-2, the second spacecraft of the Interball project, also called the Auroral Probe, is aimed at studying dissipation processes of the energy transferred to the auroral region along the open magnetic field lines from the magnetospheric tail. Some contribution to this dissipation is auroral kilometric radiation (AKR), a strongly variable radioemission occurring in the frequency range from about $30 \mathrm{kHz}$ to $1 \mathrm{MHz}$, with a maximum intensity at around $300 \mathrm{kHz}$. Spectral observations of the AKR carried from Interball-2 with POLRAD instrument generally confirm previous observations, but add also some new information to spectral structures of the AKR. They will be described and discussed in this work.

The Interball-2 spacecraft was launched into an elliptical orbit with the apogee at $19140 \mathrm{~km}$, and perigee at $780 \mathrm{~km}$, inclined to the equator for $62.8^{\circ}$, with a nodical period of $5.9 \mathrm{~h}$. It crosses the radiation belts eight times a day. For safety purposes POLRAD has been operating only outside the radiation belts, that is at $L>4.5$. With this restriction, data acquisition on AKR is being continuously performed in sessions lasting up to $4 \mathrm{~h}$ every orbit, when the spacecraft moves in the auroral region or the polar cap, at altitudes above $9000 \mathrm{~km}$, and covering invariant latitudes from $63^{\circ}$ to $80^{\circ}$.

AKR, first reported by Benediktov et al. (1965), most likely originates in the nightside auroral magnetosphere, at invariant latitudes of about $70^{\circ}$ (Gurnett, 1974), (Kurth et al., 1975), several thousand kilometres above the Earth's surface. It is mostly observed in the right handed extraordinary mode. The Viking observations (de Feraudy et al., 1987) have shown that the sources of the AKR are plasma cavities, sharp edged narrow structures, presumably field aligned with a very low plasma density. The lowest sources of the AKR were met by the low orbiting ISIS-1 satellite at $2000 \mathrm{~km}$ 
(Benson and Calvert, 1979), while the highest reported ones were crossed by Swedish Viking at about $9000 \mathrm{~km}$. This altitude can be deduced from the study of radiating and nonradiating plasma cavities made by Hilgers (1992). It has been established by Benson and Calvert (1979), James (1980), Calvert (1981), Benson (1985), and Bahnsen et al. (1989), that the AKR is generated at the local cyclotron frequency of electrons. Thus irregular and variable structures in the dynamic spectra of remote sources can be related to spatial structures of the source regions. Gurnett and Anderson (1981) interpreted the fine structures in the AKR spectra in terms of motions of the compact sources.

Observations in situ of several AKR source crossings from Viking have provided evidences that the AKR can be associated with precipitating electrons trapped in parallel electric fields of the plasma turbulence (Louarn et al., 1990). Among a number of mechanisms of the AKR generation the most widely accepted one relies on the electron cyclotron maser instability (CMI), proposed by $\mathrm{Wu}$ and Lee, (1979). It can work efficiently in density depletions, where the local plasma frequency is much lower than the electron gyro-frequency.

In this study, we present some spectral structures observed with POLRAD, characteristic by their steep cutoffs at the upper frequency limit of the AKR spectra, that, to our knowledge, have not been previously reported. We shall call these kinds of structures upper frequency cutoffs (UFCs) (Fig. 1). Occasionally, UFCs are accompanied by narrow band "ridges" of emission, at the upper frequency cutoffs (Fig. 2), which consist in enhancements of the AKR intensity by 2 orders of magnitude.

POLRAD is a swept frequency radio-spectrometer, covering the frequency range from $4 \mathrm{kHz}$ to $2 \mathrm{MHz}$ with a frequency resolution of $4 \mathrm{kHz}$. Some AKR fine structures have also been observed with MEMO, another wave experiment on board of Interball-2, operating at a lower frequency range from $5 \mathrm{~Hz}$ to $240 \mathrm{kHz}$ for short time intervals, but with much better time and frequency resolution. However, the UFCs have not been observed by MEMO, in agreement with POLRAD observations which show that they do not occur below $300 \mathrm{kHz}$.

Data from the POLRAD experiment related to the upper frequency cutoffs (UFCs) and "ridges" are described in Sect. 3. In discussion (Sect. 4) we suggest that the conditions for exciting the instability, from which the AKR originates, sharply depend on the plasma density. Above some threshold value the generation no longer operates. The instrumentation is described in Sect. 2, and conclusions are made in Sect. 5.

\section{Instrumentation}

The wave complex on board Interball-2 consists of 4 independent experiments: POLRAD, the radio-spectro-polarimeter for the frequency range from $4 \mathrm{kHz}$ to $2 \mathrm{MHz}$, MEMO the wave analyser aimed for the determination of wave characteristics and wave interac- tions in the frequency range from $5 \mathrm{~Hz}$ to $240 \mathrm{kHz}$, $\mathrm{NVK}-\mathrm{ONCH}$, the wave analyser for the frequency range from $10 \mathrm{~Hz}$ to $22 \mathrm{kHz}$, and IESP, the wave analyser working from 0.1 to $30 \mathrm{~Hz}$.

POLRAD can be used either as a radio-spectropolarimeter, or as a radio-spectrograph. For our purpose only spectrographic data have been analysed. The use of POLRAD as a polarimeter will be presented in another paper. POLRAD as a radio-spectrograph can use 1 or 3 antennae. Their coordinate system is fixed to the spinning spacecraft (1 revolution in $2 \mathrm{~min}$ ). One antenna is an $11 \mathrm{~m}$ monopole (tip-to-spacecraft centre) oriented along the spin axis in an anti-solar direction. The other antennae are 2 crossed dipoles, $22 \mathrm{~m}$ tip-totip, perpendicular to the spin axis. The size of the monopole, which is half as long as the other dipoles is compensated by the doubled voltage gain of its preamplifier. The antenna diameter is $25 \mathrm{~mm}$.

In the spectrograph mode the power spectra of signals received from each antenna are measured in separate channels. The step increment of SFA is $4.096 \mathrm{kHz}$, while the half-power bandwidth is $2.7 \mathrm{kHz}$. The step filters were made with a special care to have narrow wings, so that the power leakage between successive frequency steps is about $4 \%$. The instrumental frequency profiles of the monochromatic calibration signals measured every $4.096 \mathrm{kHz}$ by the radio-spectrograph are inserted in Figs. 1c and 2c. POLRAD can be swept over several frequency ranges selected between $4 \mathrm{kHz}$ up to $2 \mathrm{MHz}$. The frequency range most frequently used can be set from 4 to $491 \mathrm{kHz}(120$ steps), or from 4 to $983 \mathrm{kHz}$ (240 steps). The sweep rates can be set to 25,50 , or $100 \mathrm{~ms}$ per step. The resulting sweep periods, that are most frequently used, are 6 or $12 \mathrm{~s}$. The time constant of the output detector is $6 \mathrm{~ms}$. It is thus much shorter than the duration of a single frequency step. The characteristics of the filters and output detectors make a single measurement at one frequency step practically independent from that in preceding one. This favours detection of spectral structures, whose bandwidths are comparable with the frequency interval of a single step.

The sensitivity of the instrument is determined by the noise level of the antenna amplifiers. At several frequencies it is limited by onboard interferences. The latter are dominating and can be seen in the spectrograms as strong horizontal lines of almost constant amplitudes. Some of them occur at frequencies

Fig. 1a-c. The dynamic spectrum of the AKR showing upper frequency cutoffs, obtained with POLRAD on the 25th of January, 1997. a A quick look $1 \mathrm{~h}$ spectrogram received with the antenna X. Upper frequency cutoffs (UFCs) are indicated by bars above the spectrogram. Power density estimated from the colour scale should be multiplied by 100 to compensate the $20 \mathrm{~dB}$ input attenuation of the receiver. b Zoom of the same spectrogram showing the detailed spectrum of one of the UFCs in time interval from 10.41 to 10.43 UT. c Eight selected frequency profiles for the SFA sweeps indicated by numbers in the middle panel. The profiles are built up from points separated by $4.096 \mathrm{kHz}$ intervals (SFA frequency steps). For comparison the instrumental frequency profile of channel $\mathrm{X}$ is shown 


\section{EXPERIMENT POLRAD}

Antenna $X$

UT: 1997-01-25 10:41:41 - 10:42:34
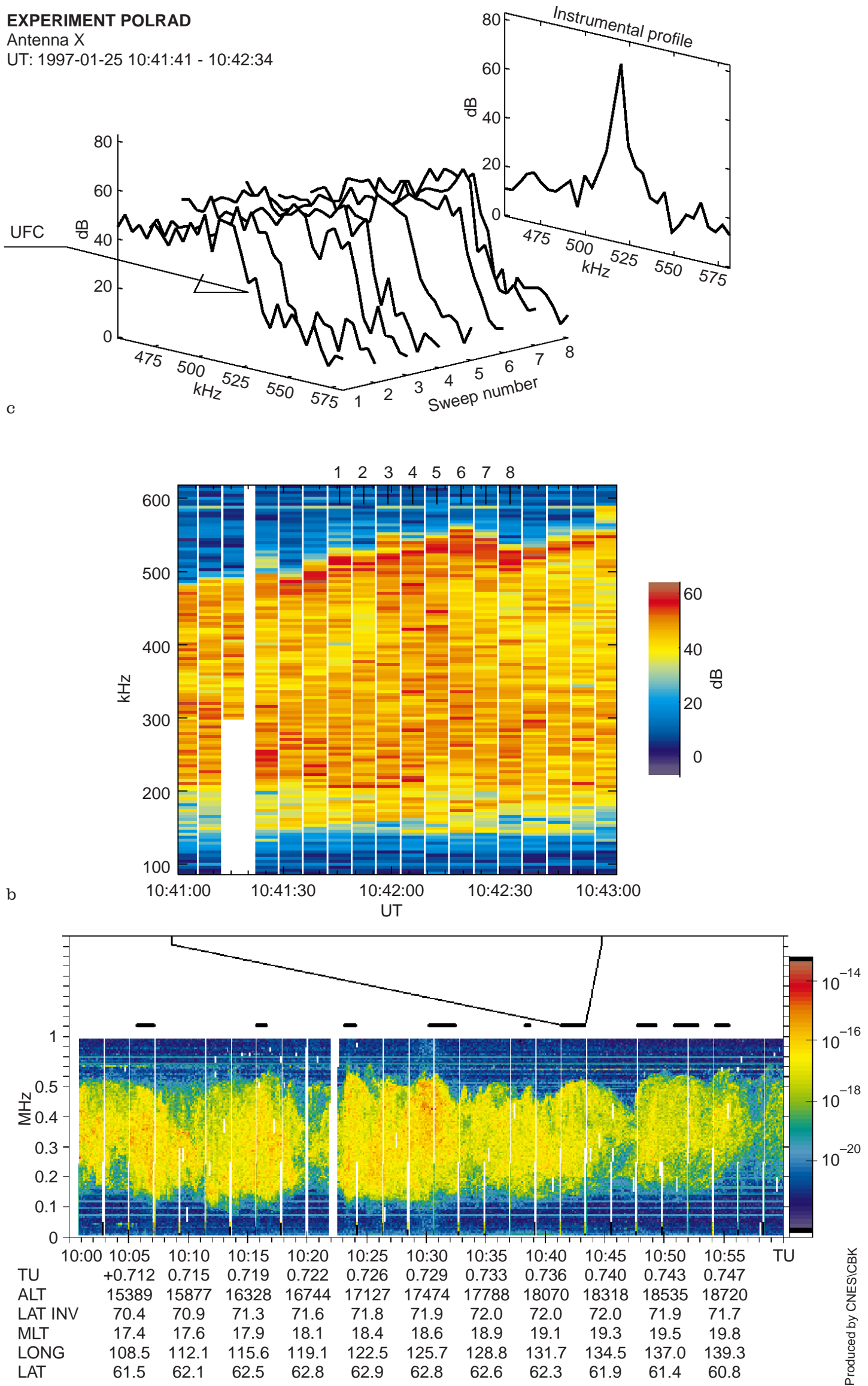

b

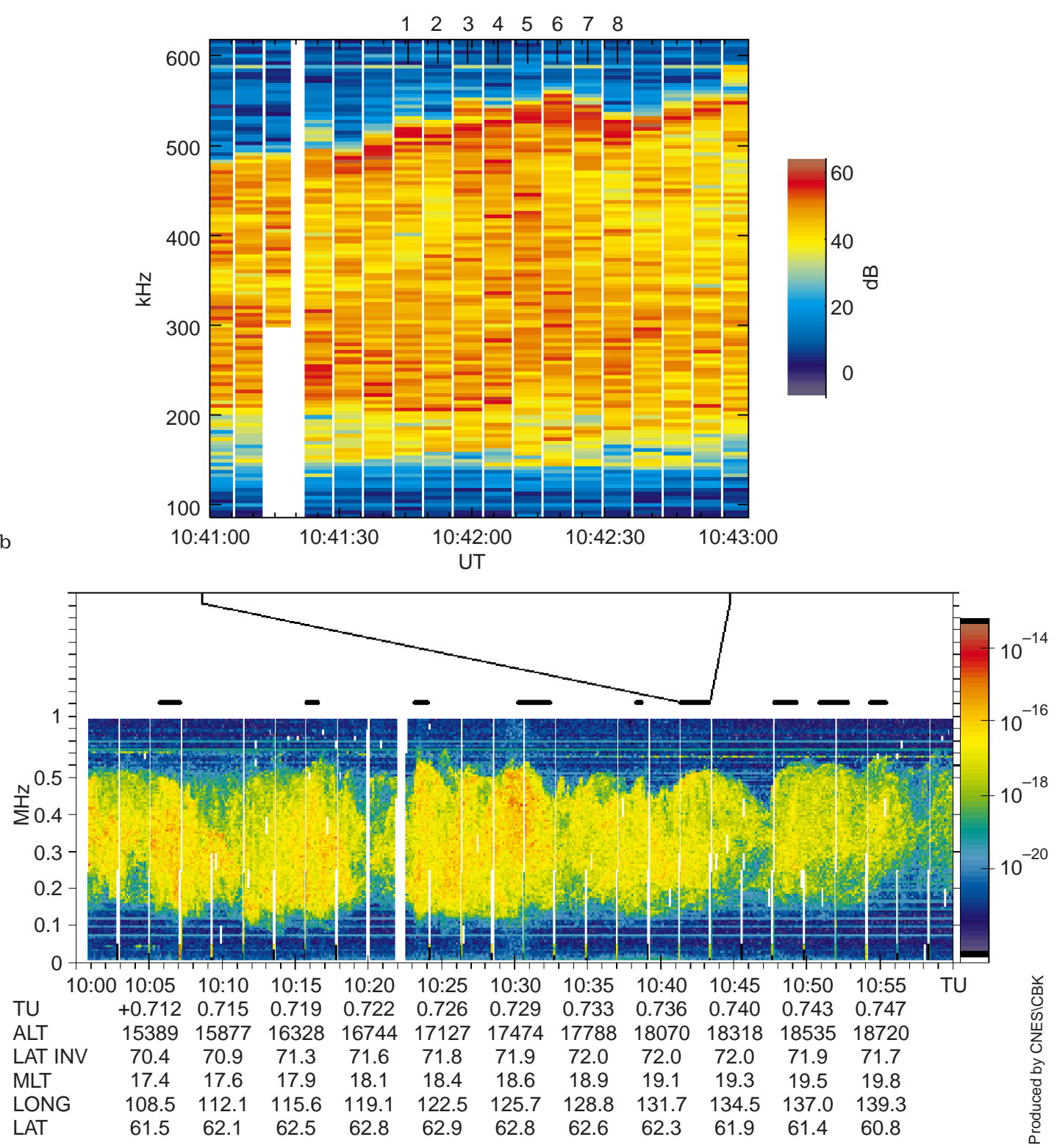

Processing data version: V3.1 - plotting data version: V3.2 - printing date: 2 June 1997 


\section{EXPERIMENT POLRAD}

\section{Antenna $Y$}

UT: 1996-10-25 14:35:20 - 14:45:00
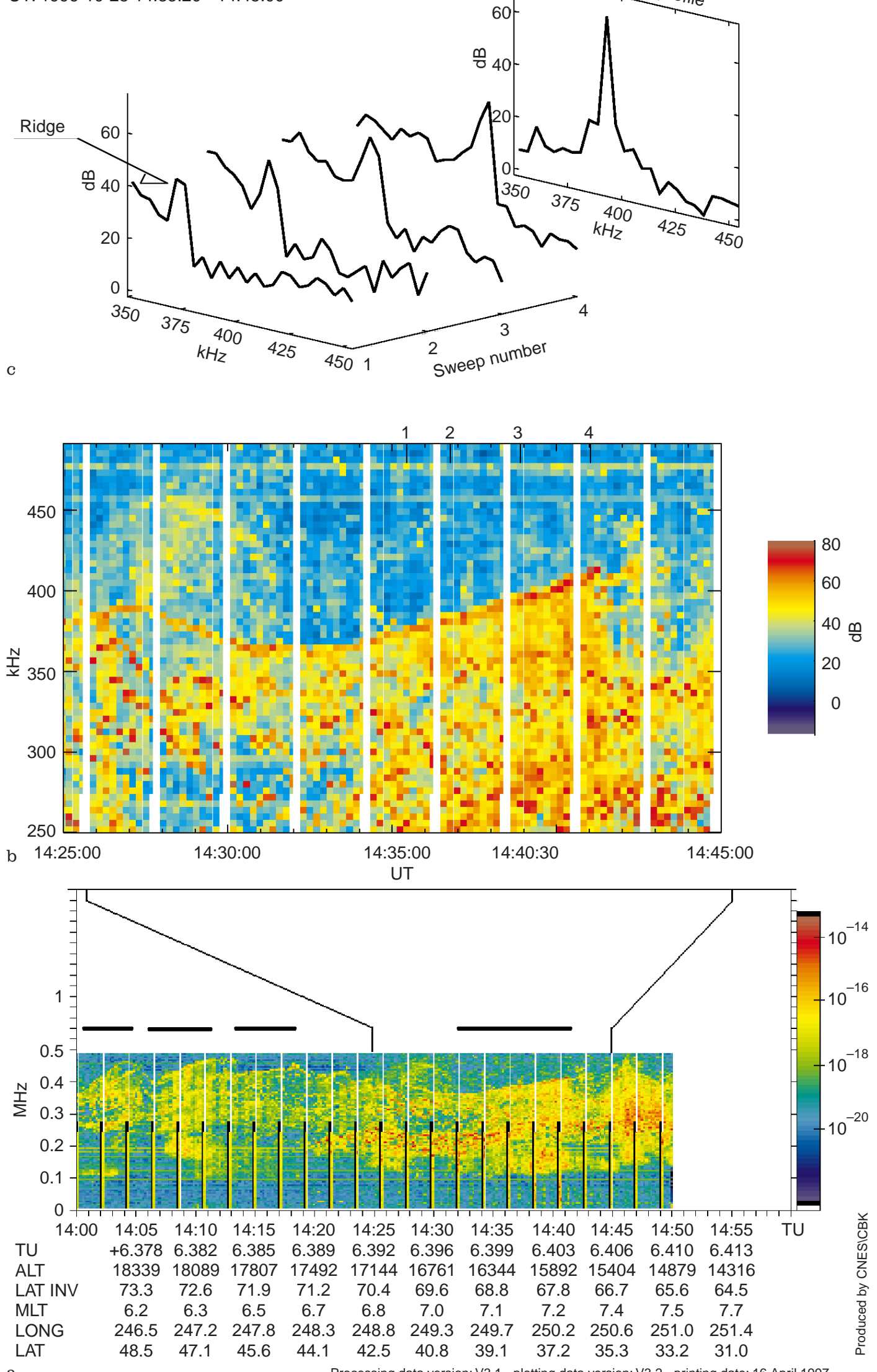

Processing data version: V3.1 - plotting data version: V3.2 - printing date: 16 April 1997 
Fig. 2a-c. The dynamic spectrum showing "ridges" at upper frequency cutoffs of AKR emission, obtained with POLRAD on the 25 th of October 1996. a A quick look at $1 \mathrm{~h}$ spectrogram received with the antenna Y. The "ridges" at upper frequency cutoffs are indicated by bars above the spectrogram. Power density can be estimated from the colour scale (no input attenuation). b Zoom of the longest "ridge" seen from 14.25 to 14.41 UT. c Four selected frequency profiles for the SFA sweeps indicated by numbers in the middle panel. The profiles are built as in Fig. 1. For comparison the instrumental frequency profile for channel $\mathrm{Y}$ is shown

$n \times 24.57 \mathrm{kHz}$. The sensitivity of the instrument is $2.5 \times 10^{-20} \mathrm{~W} /\left(\mathrm{m}^{2} \mathrm{~Hz}\right)$, if the theoretical impedance of the short dipole in vacuum is assumed. To avoid signal saturation and generation of harmonics in the wide band antenna amplifiers, the programmable input attenuation can be applied. Frequently used is the attenuation of $20 \mathrm{~dB}$, which efficiently reduces these instrumental effects. The output signal of the receiver is proportional to the logarithm of the input voltage.

The instrument has an additional active mode aimed at the determination of the frequencies of the plasma resonances, excited in the ambient plasma by a built-in relaxation sounder. Its operation interrupts the passive reception, which is seen in the spectrograms as periodic vertical gaps, every $2 \min 8 \mathrm{~s}$.

The mode of operation, sweep rate, antenna selection, frequency range, and input attenuation can be controlled from the ground. Detailed description of the equipment and POLRAD experiment can be found in Hanasz et al. (1995).

\section{Observations}

The AKR was very frequently observed with POLRAD during the first year of Interball-2 operation. Its dynamic spectra are usually smooth at upper frequencies. However, some of them demonstrated abrupt cutoffs at upper frequencies, which we call the upper frequency cutoffs (UFCs). We define the UFC as a flat spectral structure of AKR, whose intensity decreases abruptly at its upper frequency limit at least for 100 times in no more than $12 \mathrm{kHz}$ (three steps of $4 \mathrm{kHz}$ bandwidth). It may be noted that some UFCs are so steep that their frequency profiles are determined by the instrumental bandwidth of $4 \mathrm{kHz}$. The examples of UFCs are shown in Fig. 1a. It may be noticed that the AKR spectra are rather flat at frequencies below the UFC, indicating that the generation and propagation conditions do not change much for emissions at frequencies below the cutoff.

The UFCs are occasionally accompanied by narrow band enhancements of emission, which we call the "ridges" of emission, observed at the upper cutoffs (Fig. 2). Their observed bandwidths are comparable with instrumental ones. In the following subsections we shall discuss these structures separately.

\subsection{Upper frequency cutoffs}

The bottom panel (Fig. 1a) shows a quick-look spectrogram received on January 25, 1997 with the antenna $\mathrm{X}$, parallel to the spin axis of the spacecraft.

The UFCs are seen in a number of time intervals, lasting from $30 \mathrm{~s}$ to $4 \mathrm{~min}$ indicated by horizontal bars above the spectrogram.

The frequencies of single UFC structures vary over tens of $\mathrm{kHz}$. With different slopes, positive, zero and negative, the UFCs dynamic spectra are reminiscent sometimes of bell-shaped structures. In this example they occur between 430 and $650 \mathrm{kHz}$. At the same time the local gyro-frequencies of electrons, determined by the on-board magnetometer vary from 25 to $40 \mathrm{kHz}$. The UFCs must be features of the remote AKR, if generation at the gyro-frequency of electrons is accepted.

It can be concluded from review of the data received during one year of spacecraft operation, that the occurrence of the UFCs is limited to the frequency range from 300 to $700 \mathrm{kHz}$ and durations from seconds to tens of minutes.

An enlarged view of the UFCs structure is presented in Fig. 1b, in which every sweep of the SFA can be seen as a vertical strip. The period of sweep repetition is $6 \mathrm{~s}$, the step duration is $25 \mathrm{~ms}$. In this panel every single $4 \mathrm{kHz}$ step can be easily identified in order to see variations in the detailed structure of UFC. Some eight sweeps, indicated by vertical ticks have been selected to draw their frequency profiles (Fig. 1c). The mean slope (mean spectral index) of UFC is large, $6 \mathrm{~dB} / \mathrm{kHz}$, which is not much less than the instrumental one, $9 \mathrm{~dB} /$ $\mathrm{kHz}$.

We have found 515 cases of UFCs in spectrograms recorded by POLRAD in the first year of Interball-2 operation, from September 4, 1996 to October 8, 1997. The selection comprises all the cases equal to or longer than $1 \mathrm{~min}$. Most of them lasted for 1 to $5 \mathrm{~min}$, but there were some of up to $70 \mathrm{~min}$. Figure $3 \mathrm{a}$ shows distribution of spacecraft positions at times of UFC observations. One can see that most frequently the UFCs were observed when the spacecraft was around $1 \mathrm{~h}$ of magnetic local time (MLT). The number of cases decreases smoothly to 0 at noon MLT from evening and morning sides. The preponderance of cases after midnight $(0-3 \mathrm{~h}$ MLT) is clearly pronounced. Frequent occurrence of UFCs at the day side can be explained either by the existence of sources associated with UFCs at the dayside polar magnetosphere, or by transpolar propagation of radiation, facilitated by relatively wide "horizon" of the AKR visibility from the spacecraft observing mostly at apogee part of its orbit. From Fig. $3 \mathrm{~b}$ it is clear that the maximum occurrence is observed at satellite positions corresponding to the $71^{\circ}$ invariant latitude. The distribution comprises the zone of $24 \mathrm{~h}$ of MLT and invariant latitudes from $64^{\circ}$ to $80^{\circ}$ covered by orbits of Interball-2 in $1 \mathrm{y}$. It can be seen that number of cases versus invariant latitude follows the distribution of integrated times for $1 \mathrm{y}$ of POLRAD operation. The 

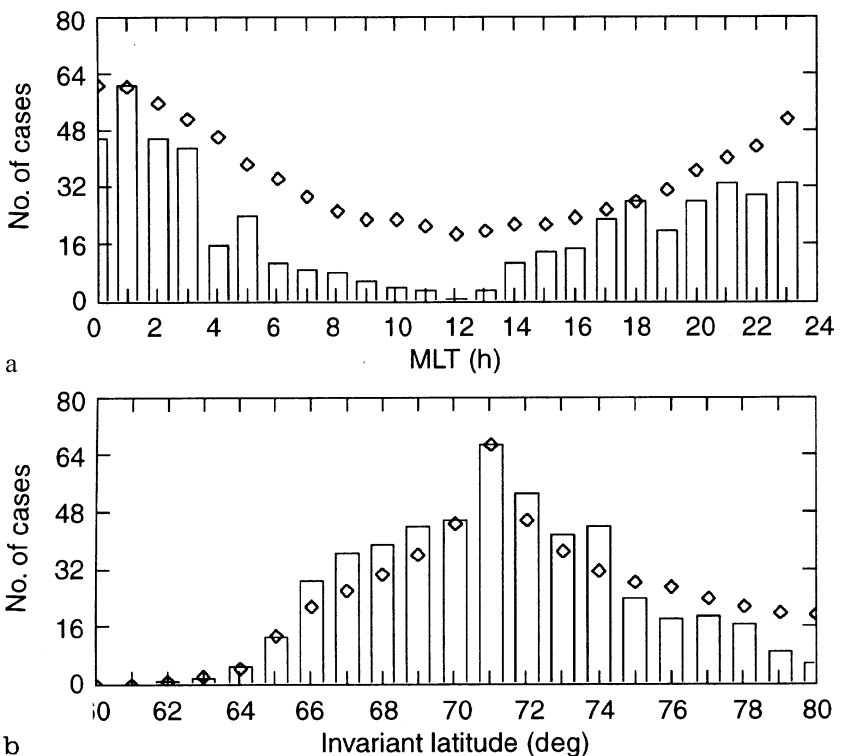

$\mathrm{b}$

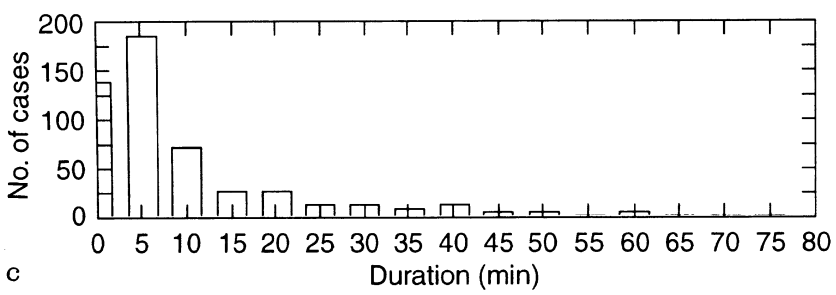

Fig. 3a-c. Statistics of 515 cases of UFCs recorded by POLRAD from September 4, 1996 to October 8, 1997. The selected cases are not shorter than 1 minute. a Distribution of spacecraft positions in MLT hours at times of UFC observations. b Distribution of spacecraft positions in invariant latitude at times of UFC observations. c Frequency of occurrence of UFC durations. On a and $\mathbf{b}$ values proportional to the integrated times of one year POLRAD operation are superposed (symbols)

distribution over MLT shows a deficiency at noon hours, which could be expected.

\section{2 "Ridges" at upper frequency cutoffs}

Filamentary fine structures of $4 \mathrm{kHz}$ bandwidth were observed frequently with POLRAD. In most cases they can be classified as narrow drifting bands already observed from other spacecraft (ISEE, JIKIKEN, DE1, Galileo) and described by Gurnett and Anderson (1981), Calvert (1982), Morioka et al. (1981) and Menietti et al. (1996). However some structures, like those shown in Fig. 2a, indicated by horizontal bars above the spectrogram, are different. They are single narrow drifting bands located at the upper cutoff frequency of the AKR, accompanied by a weaker wide emission at lower frequencies. We call them here the "ridges" at upper cutoffs.

The enlargement of the most pronounced "ridge" at UFC is shown in Fig. 2b. It can be noticed by comparison with the instrumental frequency profile, that its observed bandwidth is about $4 \mathrm{kHz}$ (1 step of SFA). It starts at about $14.25 \mathrm{UT}$ and continues to
14.41 UT, lasting for $16 \mathrm{~min}$. At the beginning this is a strong drifting downward narrow band structure, seen over the weak AKR broad band emission at higher and lower frequencies. In this phase it looks like other drifting fine structures known from previous experiments. At 14.29.10 UT the emission at frequencies below it begins to be stronger than above. At 14.31.15 UT the emission above it disappears into the receiver level. Till the end at 14.41.00 UT the "ridge" is seen at the UFC. The structure stays for 4 min at a constant frequency and at about 14.33 UT it begins to drift upward. Its intensity increases slowly together with the wide band emission below it.

In Fig. 3c we draw the line spectra of several sweeps to show examples of the frequency profiles taken at different times of the structure development. It can be noticed that in this e.g. (1) the "ridge" is stronger than the accompanying AKR background by almost $20 \mathrm{~dB}$, with its intensity increasing from 20 to $40 \mathrm{~dB}$ above the instrument noise level, and (2) the slope of the frequency profile is about $5 \mathrm{~dB} /(\mathrm{kHz})$.

There are three other "ridges" indicated by bars in this spectrogram. They are shorter and not as strong as the one described.

The series of "ridges" observed on October 25, 1996 seems to be exceptional. It must be admitted from visual inspection of quick look spectrograms, that the number of "ridges" at UFC met till now in POLRAD data is about 30, much less than the number of UFCs. Their duration can be even as short as $1 \mathrm{~min}$.

\subsection{Summary of observational results}

Now we can summarise general observational features of the upper frequency cutoff (UFC) structures:

1. The UFCs are frequently observed spectral features of the AKR, with a frequency extent of 4 to $12 \mathrm{kHz}$.

2. The UFCs are structures drifting in frequency, with a variety of patterns in the dynamic spectra, sometimes of bell-shape.

3. The UFCs are features of remote AKR. Their frequencies can vary from 300 to $700 \mathrm{kHz}$.

4. The "ridges" at UFCs are observed occasionally. They have an extent of about $4 \mathrm{kHz}$, and power densities up to 2 orders of magnitude larger than that of the simultaneous low frequency wide band emission of the AKR. The "ridges" are structures slowly drifting in frequency, which can last up to $15 \mathrm{~min}$.

\section{Discussion}

The spectrum of AKR can be considered as a superposition of contributions radiated by a chain of elementary sources extended along the auroral magnetic field lines and emitting close to the local gyro-frequencies of electrons. The cutoff at a given frequency can be related to the bottom of the source, located at an altitude determined by its local gyro-frequency of electrons. The 
frequency range of the observed UFCs, from 300 to $700 \mathrm{kHz}$, corresponds to altitudes of source bottoms from approximately 4800 to $2100 \mathrm{~km}$ for invariant latitude of $70^{\circ}$.

For the UFC width of $4 \mathrm{kHz}$ (1 SFA step) the vertical distance between strongly radiating and nonradiating levels of a source should be no more than about $20 \mathrm{~km}$ at frequency $530 \mathrm{kHz}$, corresponding to the source bottom at an altitude of around $2900 \mathrm{~km}$ (Fig. 1). From the measured spectral indices of the frequency profiles plotted in Fig. 4 the e-folding field aligned distances of amplification have been estimated to be between 3 and $8 \mathrm{~km}$.

We learn from the observations reported here that the generation process must be very sensitive to threshold conditions within the plasma depletion. It is known that the field-aligned gradient of electron density is critical for the generation mechanism. At some low altitude, when the ratio of $f_{p e} / f_{c e}$ becomes sufficiently small, the mechanism switches on at the corresponding gyro-frequency. According to the CMI theory ( $\mathrm{Wu}$ and Lee, 1979) the most intense R-X mode can be produced provided the plasma frequency is much lower than the gyro-frequency of electrons inside the source. The threshold value of $f_{p e} / f_{c e}$ was theoretically calculated by Melrose et al. (1984) to be $\leq 0.3$ for $4 \mathrm{keV}$ electrons, and was experimentally determined by Hilgers (1992) to be even $\leq 0.14$. It can be expected that such values can be met at altitudes of source bottoms determined from frequencies of UFCs. Steepness of the UFC suggests that the growth rate of emission increases rapidly when the ratio of $f_{p e} / f_{c e}$ inside the plasma depletion decreases below some threshold value. The rapid response of the generation mechanism to continuously varying electron density points towards the non-linear character of the process.

The "ridges" at first sight are similar to the AKR fine structures reported in a number of papers, see for example Gurnett and Anderson (1981), Calvert (1982), Morioka et al. (1981), Baumback and Calvert (1987),

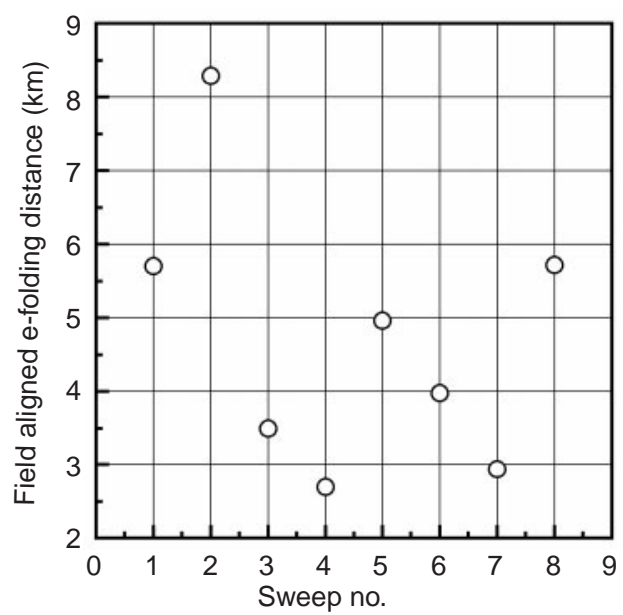

Fig. 4. The e-folding field aligned distances (expressed in kilometres) of the emission growth at the upper frequency cutoff, estimated for selected sweeps (1 to 8) of the case observed on January 25, 1997
Menietti et al. (1996). Though the idea "fine structure" includes different time and frequency scales (Menietti et al., 1996) we distinguish the "ridges" as a separate class of fine structures due to their position exactly at UFCs.

There are, in our opinion, two possible approaches to explanation of these structures: (1) in terms of a generation mechanism, and (2) in terms of propagation effects. In the first approach the "ridge" emission could be interpreted in the frame of the source bottom proposed above for UFCs as a consequence of the concentration of some amount of energy in the narrow layer just at the bottom of the source of AKR. The power density up to 100 times higher than that of the simultaneous AKR continuum, would be radiated from a thin vertical layer about $\leq 30 \mathrm{~km}$ thick, corresponding to $\leq 40$ wavelengths for frequencies around $400 \mathrm{kHz}$. However, the continuous power supply for such a longlasting (15 min) compact structure radiating high power and located at the source bottom, remains unclear.

Another possibility (still in the frame of the generation mechanism) corresponds to the narrow-band splitting type structures extensively studied for the Jovian decametric radiation by Leblanc and Rubio (1982). Like Jovian structures, "ridges" are also observed at the upper frequency limits of dynamical spectra, but they neither exhibit a split band characteristic, nor any emission band accompanied by absorption. At all times only single narrow-band emission is present. The significant differences between Jovian and terrestrial auroral conditions should also be taken into account. In a recent paper Shaposhnikov and Zaitsev (1996) explain the narrow-band splitting of Jovian emissions in terms of plasma emissions generated close to the electron density maximum of Jupiter's ionosphere, heated by strong field-aligned currents connecting Io and Jupiter. For our case we do not see analogous conditions for mechanism to work at relatively elevated heights $(400 \mathrm{kHz}$ "ridge" emission level corresponds to about $3800 \mathrm{~km}$ above Earth's surface).

In case of propagation effects ray focusing can be considered. As shown by de Feraudy and Schreiber (1995), a significant effect is possible for rays emitted slightly downwards (up to about $10^{\circ}$ with respect to the direction perpendicular to the magnetic field vector) from the source before being reflected upward. However, a persistent narrow band focusing of power density 100 times higher than the simultaneous AKR continuum, lasting for $15 \mathrm{~min}$, when the spacecraft moves over a distance of the order of a thousand kilometres, does not seem possible. As regards to the generation of "ridge" radiation discussed here, no suggestion or explanation can be proposed at the moment.

\section{Conclusions}

The following conclusions can be drawn from this study:

1. The spectral observations of the sharp upper frequency cutoffs (UFCs) of the AKR, with bandwidth from 4 to $12 \mathrm{kHz}$ are reported here, for the first time to our knowledge. 
2. UFCs occurrence is limited to frequencies from 300 to $700 \mathrm{kHz}$. It corresponds to source bottoms located at low auroral magnetosphere at altitudes around 2100 to $4800 \mathrm{~km}$.

3. From the steepness of UFCs it is suggested that the process of AKR generation is extremely sensitive to the plasma conditions and can abruptly switch on in auroral plasma depletions, when the plasma frequency becomes sufficiently low compared to the gyro-frequency of electrons. It is thus suggested that it can have a nonlinear character.

4. Very seldom $(<10 \%)$ UFCs are accompanied by narrow band "ridges" of the $4 \mathrm{kHz}$ bandwidth (instrumental) at the cutoff frequency. The intensities of the "ridges" can be up to 100 times those of the simultaneous AKR continuum. Their durations are of the order of minutes, up to $15 \mathrm{~min}$.

5. In the search for an explanation of the "ridges" at UFCs three possibilities have been briefly discussed: energy concentration at bottoms of the AKR sources, Jovian-like narrow-band split emissions, and propagation effects. No suggestions can be made about the generation mechanism, but propagation effects seem to be least possible.

6. Bandwidths of the UFCs and "ridges" are comparable with the instrumental profile of POLRAD. For study of their fine structures in more detail an instrument with better time and frequency resolution is needed.

Acknowledgements. The Polish space experiment POLRAD, carried out by the Space Research Centre P.A.S., Poland, has been developed together with the Nicolaus Copernicus Astronomical Centre P.A.S. and the Aviation Institute in Warsaw. The authors thank to the staffs of the Space Research Institute R.A.S. (Moscow) for their huge work in testing scientific equipment of the Auroral Probe, the Lavochkin Institute in Moscow for building the spacecraft, and Eupatoria Control Station (Ukraine) and Panska Ves (Czech Republic) telemetry station for reception of the experimental data. Thanks are also due to Joelle Durand and the team from CNES Toulouse for reduction and visualisation of the POLRAD data. The authors are grateful to Professor Yuri Galperin and 2 anonymous referees for constructive comments and critical remarks. This work was financially supported by the Committee of the Scientific Research in Poland (grant number 2 P03C 008 08).

Topical Editor K.-H. Glassmeier thanks L. F. Ziebell and Ph. Zarka for their help in evaluating this paper.

\section{References}

Bahnsen, A., B. Pedersen, M. Jespersen, E. Ungstrup, L. Eliason, J. Murphree, R. Elphinstone, L. Blomberg, G. Holmgren, and L. J. Zanetti, Viking observations at the source region of auroral kilometric radiation, J. Geophys. Res., 94, 6643, 1989.
Baumback, M. M., and W. Calvert, The minimum bandwidths of auroral kilometric radiation, Geophys. Res. Lett., 14, 119, 1987.

Benediktov, E. A., G. Getmantsev, Y. A. Sazonov, and A. F. Tarasov, Preliminary results of measurements of the intensity of distributed extra-terrestrial radio frequency emission at 725 and $1525 \mathrm{kHz}$ (in Russian), Kosm. Issled., 3, 614, 1965.

Benson, R. F., Auroral kilometric radiation: wave modes, harmonics and source region density structures, J. Geophys. Res., 90, 2753, 1985.

Benson, R. F., and W. Calvert, ISIS- 1 observations of the source of AKR, Geophys. Res. Lett., 6, 479, 1979.

Calvert, W., The AKR emission cone at low frequencies, Geophys. Res. Lett., 8, 1159, 1981.

Calvert, W., A feedback model for the source of auroral kilometric radiation, J. Geophys. Res., 87, 8199, 1982.

de Feraudy, H., B. Pedersen, A. Bahnsen, and M. Jespersen, Viking observations of auroral kilometric radiation from the plasmasphere to night auroral oval source regions, Geophys. Res. Lett., 14, 511, 1987.

de Feraudy, H., and R. Schreiber, Auroral radiation ray distribution in the light of Viking observations of AKR, Geophys. Res. Lett., 22, 2973, 1995.

Gurnett, D., and R. Anderson, The kilometric radio emission spectrum: relationship to auroral acceleration processes, Physics of Auroral Arc Formation, Geophys. Monogr. Ser., 25, 341, 1981.

Gurnett, D. A., The Earth as a radio source: terrestrial kilometric radiation, J. Geophys. Res., 79, 4227, 1974.

Hanasz, J., R. Schreiber, H. de Feraudy, Z. Krawczyk, K. Dudzinski, A. Krayniuk, M. Barylko, J. Juchniewicz, A. Buczkowska, T. V. Romantsova, M. M. Mogilevsky, V. N. Nazarov, and N. Mikhalev, Polrad - description of experiment, Interball mission and payload, Ed. CNES, p. 313, 1995.

Hilgers, A., The auroral plasma cavities, Geophys. Res. Lett., 19, 237, 1992.

James, H. G., Direction of arrival measurements of auroral kilometric radiation and associated ELF data from ISIS-1, J. Geophys. Res., 85, 3367, 1980.

Kurth, W. S., M. M. Baumback, and D. A. Gurnett, Direction finding measurements of auroral kilometric radiation, J. Geophys. Res., 80, 2764, 1975.

Leblanc, Y., and M. Rubio, A narrow-band splitting at the Jovian decametric cutoff frequency, Astron. Astrophys., 111, 284, 1982.

Louarn, P., A. Roux, H. de Feraudy, D. Le Quéau, M. Andre, and L. Matson, Trapped electrons as a free energy source for the auroral kilometric radiation, J. Geophys. Res., 95, 5983, 1990.

Melrose, D. B., R. G. Hewitt, and G. A. Dulk, Electron-cyclotron maser emission: relative growth and damping rates for different modes and harmonics, J. Geophys. Res., 89, 897, 1984.

Menietti, J. D., H. K. Wong, W. S. Kurth, D. A. Gurnett, L. J. Granroth, and J. B. Groene, Discrete, stimulated auroral kilometric radiation observed in the Galileo and DE1 wideband data, J. Geophys. Res., 101, 10673, 1996.

Morioka, A., H. Oya, and S. Miyatake, Terrestrial kilometric radiation observed by satellite JIKIKEN (EXOS-B), J. Geomagn. Geoelectr., 33, 37, 1981.

Shaposhnikov, V. E., and V.V Zaitsev, On the origin of the narrow-band splitting at the Jovian decametric cutoff frequency, Astron. Astrophys., 305, 352, 1996.

Wu, C. S., and L. C. Lee, A Theory of the terrestrial kilometric radiation, Astrophys. J., 230, 621, 1979. 